\title{
Chemical Constituents from Rhizomes of Curculigo breviscapa
}

\author{
Cui-Cui Zhu, Kai-Jin Wang, ${ }^{*}$ Zhi-Yuan Wang, and Ning Li* \\ Anhui Province Key Laboratory of Research and Development of Chinese Medicine, School of Life Sciences, \\ Anhui University, Hefei 230039, P. R. China. ${ }^{*}$-mail: wkjahla@sina.com(KW); ln0110@sina.com(NL) \\ Received May 29, 2009, Accepted November 22, 2009
}

Key Words: Curculigo breviscapa, Hypoxidaceae, Constituents, Breviscapin A

We have studied several species of the genus Curculigo, well known for their use in Chinese folk medicine, on their phytochemical and pharmacological characteristics. ${ }^{1-6}$ Curculigo breviscapa S. C. Chen (Hypoxidaceae) is distributed in southwest region of Guangxi Province of China. ${ }^{7}$ However, so far, the chemical constituents of this plant have not been reported. The interesting medicinal properties of this genus encouraged us to undertake the phytochemical investigation on C. breviscapa, which led to the isolation and identification of one novel phenolic compound, breviscapin A (1), together with five known compounds, crassifogenin B (2), ${ }^{2}$ crassifoside A (3), ${ }^{2}$ 3-hydro$\mathrm{xy}-5$-methylphenol-1- $O$-[ $\beta$-D-glucopyranosyl- $(1 \rightarrow 6)-\beta$-D-glucopyranoside] (4), ${ }^{8}$ ocinol glucoside (5), ${ }^{9}$ and 3,4-dihydroxybenzioc acid (6) from its rhizomes as shown in Figure 1. Their structures were established by spectroscopic analysis, especially using 2D-NMR techniques $\left({ }^{1} \mathrm{H}-{ }^{1} \mathrm{H}\right.$ COSY, HMQC, HMBC) and comparisons of their data with literature values. All the compounds in Figure 1 were isolated for the first time from this plant. Herein, the structural elucidation of a novel phenolic compound 1 was provided.

\section{Experimental}

General experimental procedures. UV-2401PC spectrometer was used to obtain the UV spectra in methanol (MeOH). IR spectra were recorded on Nexus 870-FT-IR spectrophotometer with potassium bromide ( $\mathrm{KBr}$ ) pellets. Mass spectrometry (MS) was performed on VG Autospec-3000 spectrometer and APIQSTAR-Pulsar-1 spectrometer using a positive ion model. 1D and 2D NMR spectra were measured on Bruker AM-400 spectrometer with TMS as an internal standard. Column chromatography was carried out on Sephadex LH-20 gel (25 - $100 \mu \mathrm{m}$, Pharmacia Fine Chemical Co. Ltd.). Thin layer chromatography
(TLC) was carried out on silica gel G precoated plates (Qingdao Haiyang Chemical Co. Ltd.) and spots were detected by spraying with $5 \% \mathrm{H}_{2} \mathrm{SO}_{4}$ in $\mathrm{EtOH}$ followed by heating.

Plant material. The plant material was collected in Napo, Guangxi Province, China, in August 2007 and identified by Prof. Kai-Jin Wang from the School of Life Sciences, Anhui University, where a voucher specimen (No. 20070801) was deposited.

Extraction and isolation. The air-dried and powered rhizomes of C. breviscapa $(1.3 \mathrm{~kg})$ were extracted with $85 \% \mathrm{EtOH}$ $(3 \times 7 \mathrm{~L})$ under reflux for $3 \mathrm{~h}$. The combined organic layer was concentrated in vacuo to afford a residue $(52 \mathrm{~g})$. The residue was suspended in $\mathrm{H}_{2} \mathrm{O}$ and then passed through a D101 resin column eluting sequentially with water followed by $20 \%, 40 \%, 60 \%$, $80 \%$ and $95 \%$ aqueous $\mathrm{MeOH}$. The fraction $(8.5 \mathrm{~g}$ ) eluted from $20 \% \mathrm{MeOH}$ was repeatedly purified by Sephadex LH-20 chromatography (EtOH- $\left.\mathrm{H}_{2} \mathrm{O}, 0: 1-1: 0\right)$ to yield four fractions $\left(\mathrm{A}_{1}\right.$ $\left.A_{4}\right)$. Fraction $A_{1}$ was subjected on Sephadex LH-20 chromatography $\left(\mathrm{MeOH}-\mathrm{H}_{2} \mathrm{O}, 0: 1-1: 0\right.$, then $\mathrm{EtOH}$-acetone, 1:1) to afford compounds 4 (18 mg) and 5 (13 mg). Compound 6 (17 mg) was obtained from fraction $\mathrm{A}_{2}$ by column chromatography on Sephadex LH-20 (MeOH). Compounds 1 (5 mg) and 3 (9 mg) were obtained from fraction $\mathrm{A}_{3}$ by column chromatography on Sephadex LH-20 (EtOH). The fraction $\mathrm{A}_{4}$ was subjected to chromatography on Sephadex LH-20 (EtOH) repeatedly to yield compound 2 (11 $\mathrm{mg})$.

Breviscapin A (1). Black powder, IR (KBr) $v_{\max } 3415(\mathrm{OH})$, $1676(\mathrm{C}=\mathrm{O}), 1605(\mathrm{C}=\mathrm{C}), 1519,1437,1365,1290,1263,1115$, $818,780,761 \mathrm{~cm}^{-1}$; UV (MeOH) $\lambda_{\max }(\log \varepsilon) 205$ (4.60), 283 (3.87) nm. ${ }^{1} \mathrm{H}$ - and ${ }^{13} \mathrm{C}-\mathrm{NMR}$ data see Table 1. HR-TOF-MS (positive mode) $\mathrm{m} / z 337.0679[\mathrm{M}+\mathrm{Na}]^{+}$(calcd. 337.0688 for $\left.\mathrm{C}_{17} \mathrm{H}_{14} \mathrm{O}_{6} \mathrm{Na}\right)$.

Crassifogenin B (2). Pale yellow powder, ${ }^{1} \mathrm{H}-\mathrm{NMR}(400$ MHz, acetone- $\left.d_{6}\right) \delta 6.90(1 \mathrm{H}, \mathrm{d}, J=2.8 \mathrm{~Hz}, \mathrm{H}-2), 7.09(1 \mathrm{H}, \mathrm{d}$,

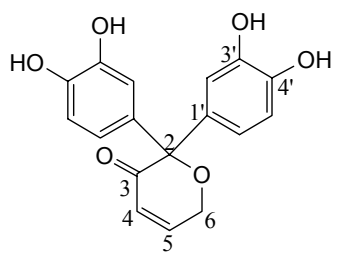

1
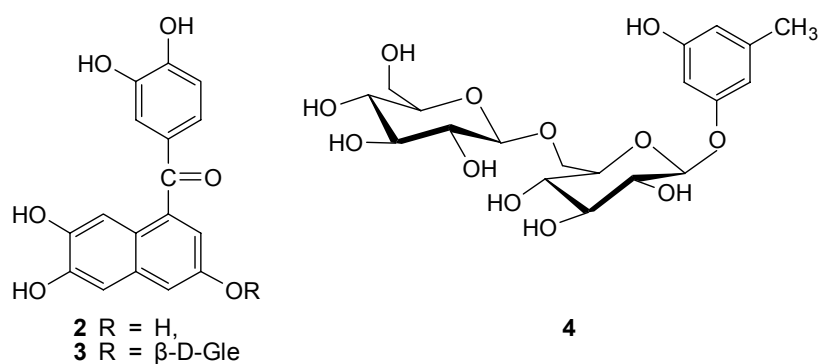
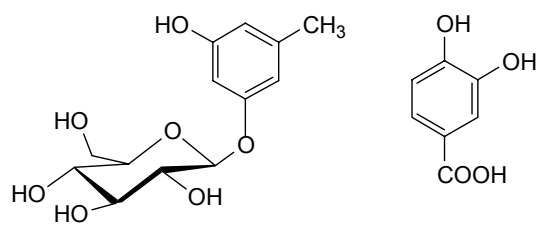

$\mathrm{COOH}$

Figure 1. Structures of compounds 1-6. 
$J=2.4 \mathrm{~Hz}, \mathrm{H}-4), 7.07(1 \mathrm{H}, \mathrm{s}, \mathrm{H}-5), 7.11(1 \mathrm{H}, \mathrm{s}, \mathrm{H}-8), 7.33(1 \mathrm{H}$, $\left.\mathrm{d}, J=2.0 \mathrm{~Hz}, \mathrm{H}-2^{\prime}\right), 6.87\left(1 \mathrm{H}, \mathrm{d}, J=8.0 \mathrm{~Hz}, \mathrm{H}-5^{\prime}\right), 7.18(1 \mathrm{H}, \mathrm{dd}$, $\left.J=8.0,2.0 \mathrm{~Hz}, \mathrm{H}-6^{\prime}\right) ;{ }^{13} \mathrm{C}-\mathrm{NMR}\left(100 \mathrm{MHz}\right.$, acetone- $\left.d_{6}\right) \delta 197.5$ $(\mathrm{s}, \mathrm{C}=\mathrm{O}), 139.0(\mathrm{~s}, \mathrm{C}-1), 117.6(\mathrm{~d}, \mathrm{C}-2), 154.0(\mathrm{~s}, \mathrm{C}-3), 112.1$ (d, C-4), 110.5 (d, C-5), 146.7 (s, C-6), 148.9 (s, C-7), 109.8 (d, C8), 123.2 (s, C-9), 133.5 (s, C-10), 132.5 (s, C-1'), 118.5 (d, C-2'), 146.3 (s, C-3'), 152.3 (s, C-4'), 116.7 (d, C-5'), 125.9 (d, C-6'); FAB-MS (+) $m / z 313[\mathrm{M}+\mathrm{H}]^{+}$.

Crassifoside A (3). White amorphous powder, ${ }^{1} \mathrm{H}-\mathrm{NMR}$ (400 $\mathrm{MHz}$, acetone- $\left.d_{6}\right) \delta 7.07(1 \mathrm{H}, \mathrm{d}, J=2.4 \mathrm{~Hz}, \mathrm{H}-2), 7.40(1 \mathrm{H}, \mathrm{d}$, $J=2.4 \mathrm{~Hz}, \mathrm{H}-4), 7.21$ (1H, s, H-5), 7.17 (1H, s, H-8), $7.34(1 \mathrm{H}$, $\left.\mathrm{d}, J=2.0 \mathrm{~Hz}, \mathrm{H}-2^{\prime}\right), 6.88\left(1 \mathrm{H}, \mathrm{d}, J=8.4 \mathrm{~Hz}, \mathrm{H}-5^{\prime}\right), 7.22(1 \mathrm{H}, \mathrm{dd}$, $J=8.4,2.0 \mathrm{~Hz}, \mathrm{H}-6$ '), 5.07 (1H, d, $\left.J=7.2 \mathrm{~Hz}, \mathrm{H}-1^{\prime \prime}\right), 3.43-3.57$ $(4 \mathrm{H}, \mathrm{m}$, glc.H), $3.69(1 \mathrm{H}, \mathrm{dd}, J=11.6,5.6 \mathrm{~Hz}, \mathrm{Ha}-6$ '), $3.90(1 \mathrm{H}$, $\left.\mathrm{dd}, J=11.6,1.6 \mathrm{~Hz}, \mathrm{Hb}-6^{\prime}\right) ;{ }^{13} \mathrm{C}-\mathrm{NMR}\left(100 \mathrm{MHz}\right.$, acetone- $\left.d_{6}\right)$ $\delta 197.5(\mathrm{~s}, \mathrm{C}=\mathrm{O}), 138.6(\mathrm{~s}, \mathrm{C}-1), 118.9(\mathrm{~d}, \mathrm{C}-2), 154.6(\mathrm{~s}, \mathrm{C}-3)$, 114.7 (d, C-4), 111.6 (d, C-5), 147.6 (s, C-6), 149.2 (s, C-7), 109.9 (d, C-8), 124.8 (s, C-9), 133.1 (s, C-10), 132.4 (s, C-1'), 118.9 (d, C-2'), 146.8 (s, C-3'), 152.7 (s, C-4'), 117.1 (d, C-5'), 126.1 (d, C-6'), 103.4 (d, C-1"), 72.6 (d, C-2"), 79.1 (d, C-3"), 75.9 (d, C-4"), 78.8 (d, C-5"), 63.9 (t, C-6"); FAB-MS (-) m/z $473[\mathrm{M}-\mathrm{H}]^{-}$

3-Hydroxy-5-methylphenol-1- $O$-[ $\boldsymbol{\beta}$-D-glucopyranosyl-( $1 \rightarrow$ 6)- $\boldsymbol{\beta}$-D-glucopyranoside (4). White amorphous powder, ${ }^{1} \mathrm{H}$ $\mathrm{NMR}\left(400 \mathrm{MHz}, \mathrm{CD}_{3} \mathrm{OD}\right) \delta 2.26\left(3 \mathrm{H}, \mathrm{s}, \mathrm{CH}_{3}\right), 6.49(1 \mathrm{H}, \mathrm{s}, \mathrm{H}-$ 2), $6.35(1 \mathrm{H}, \mathrm{s}, \mathrm{H}-4), 6.45(1 \mathrm{H}, \mathrm{s}, \mathrm{H}-6), 4.89(1 \mathrm{H}, \mathrm{d}, J=7.2 \mathrm{~Hz}$, $\mathrm{H}-1$ '), 4.46 (1H, d, $J=7.6 \mathrm{~Hz}, \mathrm{H}-1 ")$; ${ }^{13} \mathrm{C}-\mathrm{NMR}\left(100 \mathrm{MHz}, \mathrm{CD}_{3}-\right.$ OD) $\delta 21.7\left(\mathrm{q}, \mathrm{CH}_{3}\right), 160.0(\mathrm{~s}, \mathrm{C}-1), 102.0(\mathrm{~d}, \mathrm{C}-2), 159.1(\mathrm{~s}, \mathrm{C}-$ 3), 111.3 (d, C-4), 141.3 (s, C-5), 110.0 (d, C-6), 102.0 (d, C-1'), $74.8\left(\mathrm{~d}, \mathrm{C}-2^{\prime}\right), 77.8\left(\mathrm{~d}, \mathrm{C}-3^{\prime}\right), 71.3\left(\mathrm{~d}, \mathrm{C}-4^{\prime}\right), 77.3\left(\mathrm{~d}, \mathrm{C}-5^{\prime}\right), 69.6(\mathrm{t}$, C-6'), 104.5 (d, C-1"), 75.2 (d, C-2"), 77.3 (d, C-3"), 71.5 (d, C4"), 77.8 (d, C-5"), 62.6 (t, C-6"); FAB-MS (-) $m / z 447$ [M-H] .

Orcinol glucoside (5). White amorphous powder, ${ }^{1} \mathrm{H}-\mathrm{NMR}$ (400 MHz, CD $\mathrm{CD}_{3} \mathrm{OD} 2.12\left(3 \mathrm{H}, \mathrm{s}, \mathrm{CH}_{3}\right), 6.33(1 \mathrm{H}, \mathrm{s}, \mathrm{H}-2), 6.29$ $(1 \mathrm{H}, \mathrm{s}, \mathrm{H}-4), 6.21$ (1H, s, H-6), 4.76 (1H, d, $\left.J=6.8 \mathrm{~Hz}, \mathrm{H}-1^{\prime}\right)$, 3.33-3.46 (4H, m, glc.H), 3.63 (1H, dd, $J=11.6,2.4 \mathrm{~Hz}, \mathrm{Ha}-6$ '), $3.80(1 \mathrm{H}, \mathrm{d}, J=12.0 \mathrm{~Hz}, \mathrm{Hb}-6 \mathrm{\prime}) ;{ }^{13} \mathrm{C}-\mathrm{NMR}\left(100 \mathrm{MHz}, \mathrm{CD}_{3} \mathrm{OD}\right)$ $\delta 21.7\left(\mathrm{q}, \mathrm{CH}_{3}\right), 160.0$ (s, C-1), 102.2 (d, C-2), 159.1 (s, C-3), 109.8 (d, C-4), 141.2 (s, C-5), 111.2 (d, C-6), 102.1 (d, C-1'), 74.8 (d, C-2'), 77.9 (d, C-3'), 71.3 (d, C-4'), 77.9 (d, C-5'), 62.4 (t, C-6'); FAB-MS (-) $m / z 285[\mathrm{M}-\mathrm{H}]^{-}$.

3,4-Dihydroxy-benzoic acid (6). Colorless needle, ${ }^{1} \mathrm{H}-\mathrm{NMR}$ $\left(400 \mathrm{MHz}, \mathrm{CD}_{3} \mathrm{OCD}_{3}\right) \delta 7.54(1 \mathrm{H}, \mathrm{d}, J=2.0 \mathrm{~Hz}, \mathrm{H}-2), 6.90(1 \mathrm{H}$, $\mathrm{d}, J=8.4 \mathrm{~Hz}, \mathrm{H}-5), 7.48(1 \mathrm{H}, \mathrm{dd}, J=8.4,2.0 \mathrm{~Hz}, \mathrm{H}-6) ;{ }^{13} \mathrm{C}-\mathrm{NMR}$ $\left(100 \mathrm{MHz}, \mathrm{CD}_{3} \mathrm{OCD}_{3}\right) \delta 169.0(\mathrm{~s}, \mathrm{C}=\mathrm{O}), 124.1(\mathrm{~s}, \mathrm{C}-1), 118.4$ (d, C-2), 146.4 (s, C-3), 151.6 (s, C-4), 116.6 (d, C-5), 124.6 (d, C-6); EI-MS $m / z 154$ [M] $]^{+}$.

\section{Results and Discussion}

Breviscapin A (1) was obtained as black powder. Its molecular formula was determined as $\mathrm{C}_{17} \mathrm{H}_{14} \mathrm{O}_{6}$, with 11 degrees of unsaturation, on the basis of ${ }^{13} \mathrm{C}$-NMR (DEPT) data and the pseudomolecular ion $[\mathrm{M}+\mathrm{Na}]^{+}$at $m / z 337.0679$ in $\mathrm{HR}_{-} \mathrm{TOF}^{+}$-MS (calc. 337.0688). The IR spectrum indicated absorptions of $\mathrm{OH}$ groups at 3415, a carbonyl group at 1676 and a conjugated olefinic bond at $1605 \mathrm{~cm}^{-1}$. The carbonyl and olefinic IR bands were
Table 1. ${ }^{1} \mathrm{H}-\mathrm{NMR}$ and ${ }^{13} \mathrm{C}-\mathrm{NMR}$ Data of $1\left(400 \mathrm{MHz}\right.$, in $\mathrm{CD}_{3} \mathrm{OCD}_{3}, \delta$ ppm, $J$ in $\mathrm{Hz}$ )

\begin{tabular}{crll}
\hline position & \multicolumn{1}{c}{$\delta(\mathrm{C})$} & \multicolumn{1}{c}{$\delta(\mathrm{H})$} & $\mathrm{HMBC}(\mathrm{H} \rightarrow \mathrm{C})$ \\
\hline 2 & $88.3(s)$ & & \\
3 & $196.2(s)$ & & \\
4 & $128.1(d)$ & $6.14(d t, J=10.4,2.4)$ & $\mathrm{C}-2, \mathrm{C}-6$ \\
5 & $150.2(d)$ & $7.13(d t, J=10.4,2.8)$ & $\mathrm{C}-3, \mathrm{C}-4, \mathrm{C}-6$ \\
6 & $62.8(t)$ & $4.34(t, J=2.4)$ & $\mathrm{C}-2, \mathrm{C}-3, \mathrm{C}-4, \mathrm{C}-5$ \\
$1^{\prime}$ & $133.5(s)$ & & \\
$2^{\prime}$ & $117.3(d)$ & $6.72(d, J=2.0)$ & $\mathrm{C}-2, \mathrm{C}-4^{\prime}, \mathrm{C}-6^{\prime}$ \\
$3^{\prime}$ & $146.2(s)$ & & \\
$4^{\prime}$ & $146.6(s)$ & & \\
$5^{\prime}$ & $116.2(d)$ & $6.76(d, J=8.0)$ & $\mathrm{C}-1^{\prime}, \mathrm{C}-3^{\prime}$ \\
$6^{\prime}$ & $121.3(d)$ & $6.60(d d, J=8.0,2.0)$ & $\mathrm{C}-1, \mathrm{C}-2^{\prime}, \mathrm{C}-4^{\prime}$ \\
$1^{\prime \prime}$ & $133.5(s)$ & & \\
$2^{\prime \prime}$ & $117.3(d)$ & $6.72(d, J=2.0)$ & $\mathrm{C}-2, \mathrm{C}-4^{\prime}, \mathrm{C}-6^{\prime}$ \\
$3^{\prime \prime}$ & $146.2(s)$ & & \\
$4^{\prime \prime}$ & $146.6(s)$ & & $\mathrm{C}-1^{\prime}, \mathrm{C}-3^{\prime}$ \\
$5^{\prime \prime}$ & $116.2(d)$ & $6.76(d, J=8.0)$ & $\mathrm{C}-1, \mathrm{C}-2^{\prime}, \mathrm{C}-4^{\prime}$ \\
$6^{\prime \prime}$ & $121.3(d)$ & $6.60(d d, J=8.0,2.0)$ &
\end{tabular}

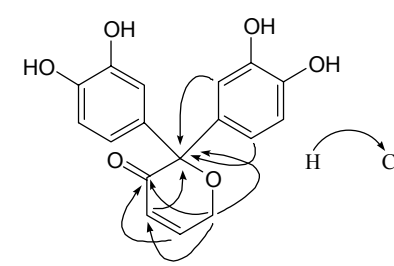

Figure 2. Key HMBC correlations of $\mathbf{1}$.

confirmed by the ${ }^{13} \mathrm{C}-\mathrm{NMR}$ signals at $\delta_{\mathrm{C}} 196.2,128.1$ and 150.2 ppm (Table 1). The ${ }^{1} \mathrm{H}-\mathrm{NMR}$ spectrum showed the presence of two symmetrical 3,4-disubstituted aromatic rings. 1D-NMR and 2D-NMR ( ${ }^{1} \mathrm{H}-{ }^{1} \mathrm{H}$ COSY, HMQC, HMBC) experiments suggested the presence of a six-membered ring containing oxygen and $\alpha, \beta$-unsatuated ketone. Thus, the structure of 1 was elucidated as 2,2-bis(3,4-dihydroxyphenyl)-2H-pyran-3(6H)-one based on further analysis of 2D-NMR experiments.

The ${ }^{1} \mathrm{H}-{ }^{1} \mathrm{H}$ COSY correlations of $\mathrm{H}-4 / \mathrm{H}-5$, and $\mathrm{H}-5 / \mathrm{H}-6$ showed the connectivity of $\mathrm{C}(4)-\mathrm{C}(5)-\mathrm{C}(6)$. The linkage of $\mathrm{C}-2$ and $\mathrm{C}-6$ to an $\mathrm{O}$-atom was established by the HMBC correlations of $\mathrm{H}-6 / \mathrm{C}-2$ as shown in Figure 2 and the low-field chemical shifts of C-2 and C-6 at $\delta_{\mathrm{C}} 88.3$ and $62.8 \mathrm{ppm}$, respectively (Table 1). The HMBC correlations of H-5/C-3 and H-4/C-2 indicated the presence of $\mathrm{C}(4)-\mathrm{C}(3)-\mathrm{C}(2)$. Thus, a six-membered ring containing oxygen and $\alpha, \beta$-unsatuated ketone was established. The HMBC experiments also showed the long-range couplings of $\mathrm{H}-2^{\prime} / \mathrm{C}-2$ and $\mathrm{H}-6^{\prime} / \mathrm{C}-2$, which suggested that two symmetrical 3,4-disubstituted aromatic rings were connected with C-2, respectively (Figure 2). Acetylation of 1 with $\mathrm{Ac}_{2} \mathrm{O}$ in pyridine led to the preparation of tetraacetyl analogue, which indicated that four free $\mathrm{OH}$ groups are present in $\mathbf{1}$. This was confirmed by its FAB-MS spectrum showing a peak at $\mathrm{m} / \mathrm{z} 483$ corresponding to the analogue's protonated ion peak.

Five known compounds, crassifogenin B (2), ${ }^{2}$ crassifoside A 
(3), ${ }^{2}$ 3-hydroxy-5-methylphenol-1- $O$-[ $\beta$-D-glucopyranosyl-(1 $\rightarrow 6)$ - $\beta$-D-glucopyranoside] (4), ${ }^{8}$ ocinol glucoside (5), ${ }^{9}$ and 3,4dihydroxy-benzioc acid (6), were also isolated from the Rhizomes of $C$. breviscapa. Their structures were elucidated by spectral data and their comparison with literature values. All of these compounds were isolated for the first time from this plant.

Acknowledgments. This work was supported by the National Natural Science Foundation of China (30670217), International Foundation for Science (F/4340-1), the Science and Technology Foundation of Distinguished Young Scholars of Anhui Province (08040106812) and the Foundation of personnel developing of Anhui Province. (2008Z020). The authors are grateful to the staff of the Modern Experiment Technology Center, Anhui University for the spectral data.

\section{References}

1. Li, N.; Jia, A. Q.; Liu, Y. Q.; Zhou, J. Acta Bot. Yunna. 2003, 25, 241

2. Li, N.; Chen, J. J.; Zhou, J. Helv. Chim. Acta 2004, 87, 845.

3. Li, N.; Chen, J. J.; Zhao, Y. X.; Zhou, J. J. Asian Nat. Prod. Res. 2005, 7, 189.

4. Li, N.; Wang, K. J.; Chen, J. J.; Zhou, J. Tetrahedron Lett. 2005, $46,6445$.

5. Li, N.; Chen, J. J.; Zhou, J. Z. Naturforsch. B 2006, 61b, 611.

6. Wang, K. J.; Li, N. Arch. Pharm. Res. 2008, 31, 1313.

7. Delectis Florae Reipublicae Popularis Sinicae Agendae Academiae Sinicae Edita. Flora Reipublaris Sinicae; Science Press: Beijing, 1985; Vol.16, p 33

8. Roberto, R. G.; Lin, L. Z.; Geoffrey, A.; Coraell, S. Phytochemistry 1995, 39, 405 .

9. Xu, J. P.; Dong, Q. Y. Chinese Traditional and Herbal Drugs 1986, 17,8 . 